\title{
Patienten-App mit dem Health Media Award 2013 ausgezeichnet
}

Straumann überzeugte die 12-köpfige Jury des Health Media Awards mit der neuen Patienten-App fürs iPad und erhielt die gefragte Auszeichnung in der Kategorie „Patientenkommunikation“. Am 28. Juni 2013 wurde im historischen Kölner Wasserturm der Preis an Straumann überreicht. Einmal jährlich werden bei diesem Wettbewerb die Bestleistungen im Bereich der Gesundheitskommunikation prämiert und die Arbeiten einer breiten Öffentlichkeit präsentiert. Der Organisator, die Health Media Award UG, verfolgt nach eigener Darstellung das Ziel, die Kommunikation im stetig wachsenden und immer bedeutsamer werdenden Health-CareMarkt zu fördern. In diesem Jahr stand der Health Media Award unter der Schirmherrschaft des deutschen Gesundheitsministers Daniel Bahr.

„Der Preis ist für uns die Bestätigung, dass es derzeit um mehr geht, als nur

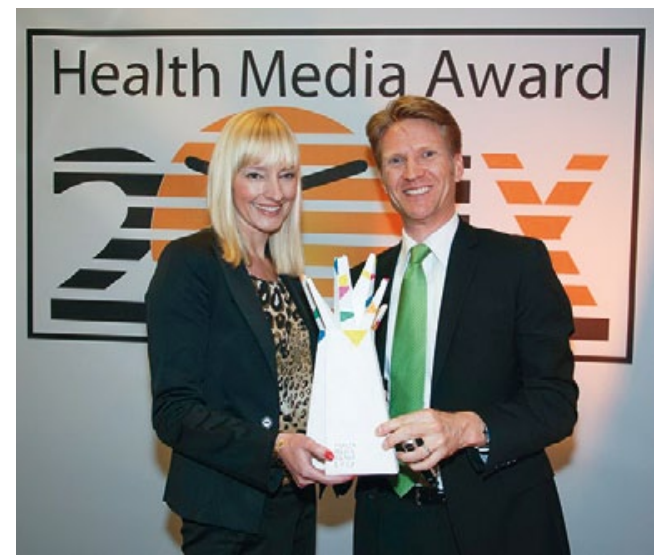

Manuela Gallus (Leiterin Marketing Communication, Straumann Deutschland) und Bernd Mahlmann (Leiter Value Added Customer Service, Institut Straumann AG, Basel) mit dem Health Media Award.

gute Produkte zu liefern. Mit unserem Kundenprogramm ,More Than Implants' haben wir schon recht früh diesen Weg eingeschlagen“, erklärt man hierzu bei Straumann. „Die App verdeutliche, welche negativen Auswirkungen fehlende
Zähne haben könnten, dass ein Zahnimplantat ein guter Ersatz für einen natürlichen Zahn sein kann und welche besonderen Vorteile Zahnimplantate gegenüber alternativen konventionellen Lösungen bieten. Dazu hätte man die Möglichkeit, gemeinsam mit dem Patienten Videos zu Behandlungsoptionen anzuschauen oder mit dem interaktiven Zeichnungstool Inhalte zu kommentieren oder hervorzuheben. Anschließend ließe sich das Gezeigte ausdrucken oder mit der integrierten E-Mail-Funktion verschicken. Alle Präsentationen landen zum Schluss in einem Archiv, aus dem sie später wieder abgerufen, erneut gezeigt und geändert werden können.“ Die Straumann Patienten-App steht den Straumann-Kunden im App-Store kostenlos zur Verfügung

Nach einer Pressemitteilung der Straumann GmbH, Freiburg Internet: www.straumann.de

\section{Erstmals mit „Sonderpreis Praxis“ ausgeschrieben}

Die Gesundheitsinitiative Wrigley Oral Healthcare Program (WOHP) stiftet auch für 2014 wieder den mit $10000 €$ dotierten Wrigley Prophylaxe-Preis. Wissenschaftler und Praktiker, die sich mit der Prävention oraler Erkrankungen oder den Zusammenhängen zwischen Mundund Allgemeingesundheit befassen, sind zur Bewerbung aufgerufen.

20 Jahre nach der Erstausschreibung gibt es nun mit der Auslobung des Sonderpreises „Niedergelassene Praxis und gesellschaftliches Engagement" ein Novum. Hierzu DGZ-Präsident Professor Roland Frankenberger aus Marburg: „Mit dem Sonderpreis sollen Menschen ausgezeichnet werden, die sich auf vielfältige Weise auch in kleineren, nicht wissenschaftlich ausgerichteten Projekten für die Verbesserung der Zahngesundheit in der Bevölkerung einsetzen.“ Prämiert werden innovative Materialien, Ideen und Konzepte aus der Zahnarztpraxis, Schule, Kindergärten, Betrieben und anderen Institutionen. Um

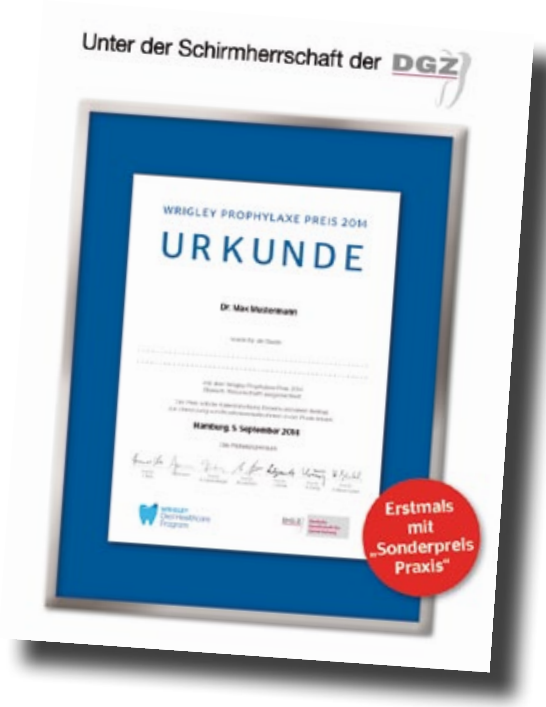

den Sonderpreis bewerben können sich Praxisteam-Mitarbeiter, Lehrer, Pädagogen und alle, die sich - auch ehrenamtlich - für die Oralprophylaxe engagieren.

Teilnahmeberechtigt sind angehende und approbierte Ärzte, Wissenschaftler und Akademiker aller Fachrichtungen unabhängig davon, ob sie in der universitären Forschung, in der Praxis oder im öffentlichen Gesundheitswesen tätig sind. Verliehen wird die Auszeichnung für neue Erkenntnisse im Bereich der Forschung und der Umsetzung der zahnmedizinischen Prävention. Das Auswahlverfahren wird von einer unabhängigen Expertenjury geleitet, die sich aus Professoren der Zahnerhaltungskunde, dem Präsidenten der DGZ sowie einem Vertreter der gesetzlichen Krankenkassen zusammensetzt.

Informationsflyer und Bewerbungsformulare mit Teilnahmebedingungen auch zum „Sonderpreis Praxis“ - sind unter www.wrigley-dental.de abrufbar oder bei kommed Dr. Bethcke, Fax: 089/33 0364 03, info@kommed-bethcke. de erhältlich. Bewerbungen sind bis zum 1. März 2014 willkommen.

Nach einer Pressemitteilung der Wrigley $\mathrm{GmbH}$, Unterhaching

Internet: www.wrigley-dental.de 